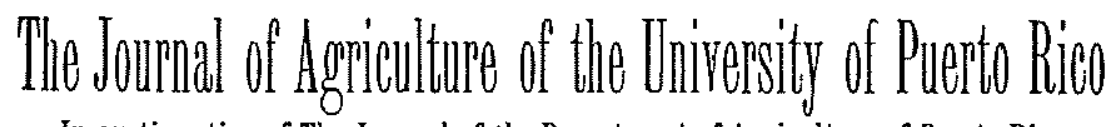

In continuation of The Journal of the Department of Agriculture of Puerto Rico Published by The Aamicultural Experiarent Station, Rio Piedras, P. R.

Pablighed Qusterly: Jannary, April, July and October of each yosr.

Mrevixte T. Cook, IExron

Vor. XXI

OCTOBER 1937

No. 4

\title{
TWO EARLY PUERTO RICAN HERPETOLOGISTS
}

\author{
Ghapana Granti, U. S. Army, Retired.
}

\section{Dr. Augustín Stahl}

Augustin Stahl was born in Aguadilla, Puerto Rico, in 1842, the son of a German father and a Dutch mother. He graduated from Wurtzburg as an M.D. in 1864 and resided in Bayamón from 1865 until his death in 1917.

Dr. Stahl's passion was natural history. Most of his work was in botany or ethnology. Fis collection of fauna numbered nearly four thousands specimens, on which he wrote four volumes in the nature of a catalogue. Herpetology was not his strong forte, as is evident by the scant space given it in his works. His greater interest in other branches cloubtless accounts for his not marking the wealth of unclassified forms about him.

Dr. Stahl is kindly remembered in Puerto Rico, as well as by scientists of international fame who have named species of many classes in his honor. Unfortunately his reptile collection, together with his correspondence with Gundlach and other herpetologists is lost. He did not describe any new species, but was the first to record Caretta caretta from Puerto Rican waters, the twenty-fifth species listed of the fifty-two now known to occur on Puerto Rico or contiguous thereto.

The inaccessibility of Dr. Stahl's books pointed to the advisability of making the appropriate parts available to interested herpetologists. This is done gratefully with the memory of two delightful years of herpetological study in Puerto Rico following in the footsteps of Augustin Stahl, the pioneer; Francisco del Valle Atiles, the tolerant and Leonhard Stejneger, the scholar.

A comprehensive appreciation of Dr. Stahl was written by Chancellor Carlos E. Chardón in "La Revista de Agricultura de Puerto Rico" 12:65-84, 1924.

We turn to Dr. Stahl's work and find on-

Pages 5-9 the "Advertencia"; a pertinent part revealing the noble character of the modest pioneer reads: 
“... ofrecer una clasificación de los animales de Puerto Rico para facilitar el conocimiento de ellos á los aficionados, despertar en la juventud estudiosa por medio de este libro la afición á las investigaciones en el inagotable campo de la Historia Natural, y en particular de nuestra fauna, iniciándoles en estudios apenas practicados en el país por falta de una guía práctica, la que pretendemos ofrecer en este trabajo, primero y único en su clase en Puerto Rico.",

"Indudablemente que la obra ha de resentirse de los defectos propios á todo trabajo que por primera vez se ejecuta: el tiempo traerá otros más competentes que se encargarán de rectificar y completar nuestra obra imperfecta.',

Bayamón, Febrero de 1882. Dr. A. Stahl.

Page 23-

“El Dr. Guillermo Peters, director del Museo zoológico de Berlín, dá á conocer en Sitzung der physikalisch mathematischen Klasse del 13 de Noviembre de 1876 inserto en la obra que se lee en Autores los mamíferos y reptiles que le fueron enviados por el Sr. Krug ... Los reptiles, que me son todos conocidos, á excepción del Anotis Krugii, dejan un vacío por la falta de los testudinados marinos. Posteriormente he encontrado una nueva especie de los Geckones, y hecho estudios más detenidos sobre la embiología del Coquí, Hylodes martinicensis, que he remitido á Berlín.", *

The ". . estudios - - sobre la embiología del Coqui . ." was first written up by Bello y Espinoza en 1871.

Page 36-

CuAdro sinóptico de las 12 clases del reino animal, dividido en tres grupos... B. Sangre fría; corazón con un ventrículo y una ó dos aurículas. Huevos casi siempre coriaceos; con 6 sin metamórfosis; respiración pulmonar, en los anfibios branquial en la primera edad; desnudos ó escamosos; 4662 ó ningunas extremidades (Coquí, Iguana)... III. Reptiles. (Reptilia)',.

Page 68: Notes-The page should commence:

"Ord. I. Testudinados (Testudinata).", to conform to the rest of the text. Line 6: "Morrocollo", The tortoise mentioned here is probably the last time it appears in literature of Antillean herpetology until the July 1932, Jour. Dept. Agri., P. R. H. West mentions its oceurrence as early as 1793. It is now found feral on several keys of the Virgin Island group and has as much right to a place in Antillean herpetology as Iguana which is supposed to have been introduced in pre-Columbian times by the Caribs for food. The word "morrocollo", means latrine cleaner; this reptile was kept for this purpose. Line 7; "Costa Firme" refers to the North Coast of Venezuela and Columbia. Line 25; "Es pequeña", referring to $C$. caretta must relate to a specific small specimen in Dr. Stahl's collection. Page 70 ; Notes; Line 28; "Culebra sabanera (Dromicus).', Dr. Stejneger named this species Leimadophis stahti in 1904 with the remark: "Dedicated to Dr. A. Stahl, of Bayamón, the venerable Porto Rican patriot and naturalist, from whom I received the type specimen.',

* Notes: The "Geckones" mentioned is Hemidactylus brookii. The exact species was not known until 1932. See "The Hemidactyls of the Porto Rico Region" Jour. Dept. Agri. P. R. Vol. XVI, No. 1. 


\section{CATÁLOGOO}

DEL

\section{GABINETE ZOOLÓGICO}

DEL

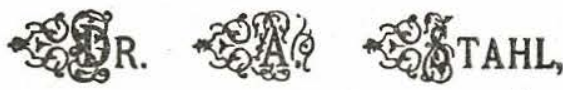

EN BAYAMON (Pto.-Rico.)

Precedido de una elasificación sistemática

de los animales que corresponden á esta fauna.

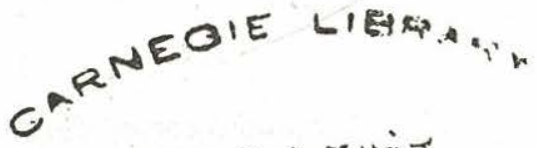

PUERTO-RICO.

Jmprenta del "Boletin Mrrcantil,"

$$
\text { 37. - Fortaleza-37. }
$$

1882 . 


\section{III. REP'TILES. (REPTilia).}

\section{Dirision de los Reptiles en cnaturo órdeness.}

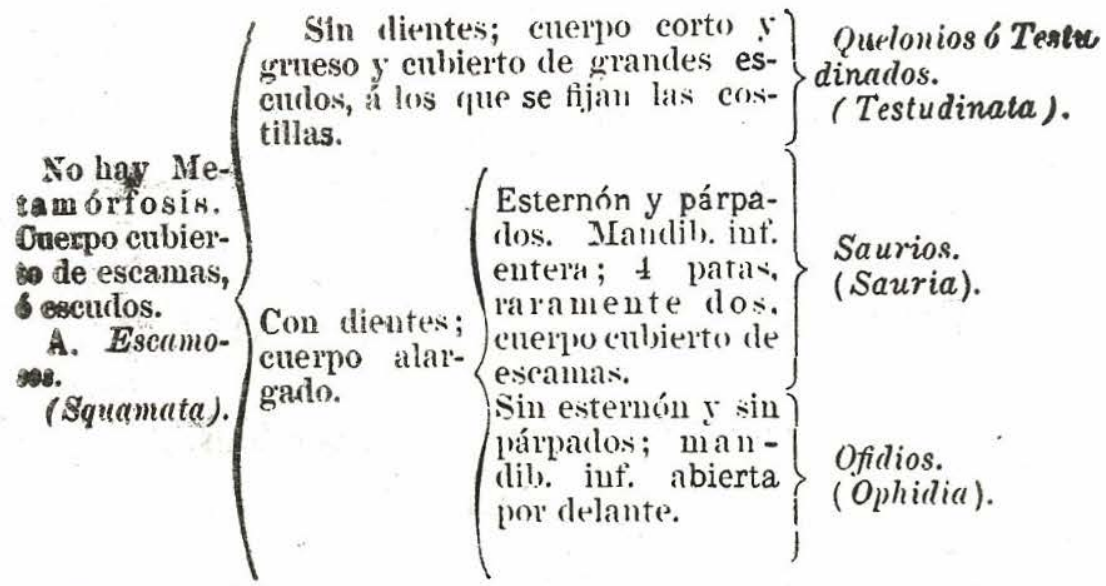

Metamorfosis. Cherpo alargado ó corto, desnudo, piel glutiuosa extremidades 40.26 uingiumas. Batráquios ó Anfibros. (Batrachia) 8. Desnudos, (Sudu.)

A. Reptias escinusos. (Reptilia squamata).

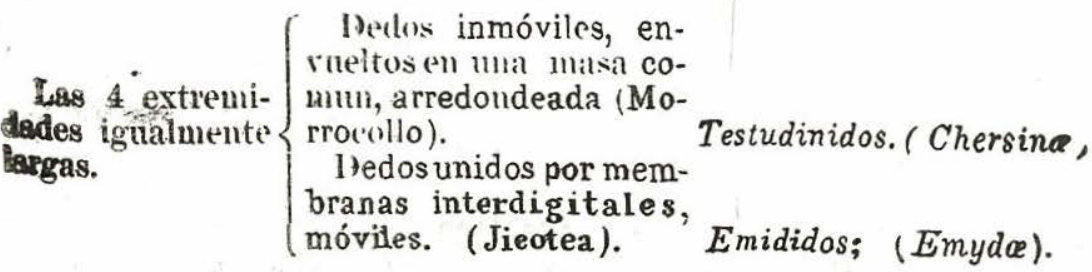




\section{(68)}

Extremidades anteriores más largas que las posteriores; dedos en forma de remos. (Carey). Quelonidos. (Chelona).

FF. 1a Testumarndos. (Chersina). Dedos envueltos en una masa comun y arredondeada, y se distingueu al exterior por las tunas; espaldar convexo y vientre en los machos con una depresion.

Morrocollo. Testudo labulata, imbricate y carbonaria, son indigenas de las sabanas de Costa Firme y propagalos en todas las Antillas.

Fu. 2a Exrordos. (Emida). Dedos unidos poy membranas interdigitales y provistos te unas largas; espaldar poco covexo.

Jicotea. Emys rugosa (decussala), Sharv. Oscura con los escudos algo verdosos con estrias y puntos amarillosos; parte inferior aularilla.

Fr. 3. QueLonmos. (Chelona). Extremidades torácicas mayores que las abdominales, todas en forma de remos, con los dedos ocultos bajo la piel; cabeza y extremidades no retractiles. Habiena ol mar.

A. Cuerpo revestido de grandes placas córneas; dedos con uñas.

Tortuga. Chelonia viridis, Schn. Verde oscura, parte inferior amarillosa, carapacho algo comprimido y elevado el dorso. Su concha poco estimada.

Carey. Ch. imbricuta, L. 13 placas variadas de amarillo y oscatro y concha muy estimada.

Cagruama. Ch. caretta, L. 15 placas provistas de unti quilla longritudinal en la primera edad. Es pequeña.

B. Cubierta del cuerpo coriácea; dedos sin uñas.

Tinglado. Sphargis coriacal, L. Color osciro con 5 quillas longitudinales en el dorso. Muy grande. En la playa del Dorado, we vio salir un tinglalo del mar, cavar un hoyo en un instante y rlepositar en él en pocos momentos setenta y oohp huevos.

Ord. 29 Saurios. (Suaria). Cuerpo alaygado, cubierto de escumas 6 escudetes; cuatro extremidades, á veces faltan dos 6 todas clas; ámbas mandíbulas y á veces tambien el paladar provistos de dientes; la uandíbula inferior se articula con el crúnco mediante el hueso timpánico; costillas movibles, esternón bien desarrollado en casi todas las especies; dos 6 tres jarpados, tímpano visible; 6 veces vejiga urinaria y pene doble casi siempre. Este brolen ol polinorfo en stus especies y presenta algumas analogías con los ofidiōn, mús que tow demás órlenes; los cocodrilos se aproximan á los mamiferos, los antibenidos á los ofidios, el Platydactylus á los sapos. Se alimentan de insectos y otros animales pequeños; son temibles las grandes especies, hs pequeñas inofensivas, careciendo todas de dientes ponzonosos ó vejiga venenosa. 7 familicts.

19 Cuerpo cubierto de escudetes y escamas de gran consisteneia. (Cocodrilo). Cocodrilos. (Crocodiline).

29 Cuerpo cubierto de encamas 6 verrugas. 


\section{(69)}

a. Lengua protractil.

1. Lengua delgada y bífida, dedos desiguales en las 4 extremidsdes. (Lagartija).

Lacertidos. (Lacertini).

2. Glutinosa gruesa en la punta, 5 dedos iguales, cola prensil.

Camaleonidos. (Chamaleonidei).

b. Lengua corta. 1. Gruesa, verrugosa, no protractil; 4 extremidades siempre.

Iguenidos. (Iguenoidei).

2. Esscotada en la punta; extremidades 4 ó 2 ó ningunas.

Escincidos. (Ścincoidei).

3. Escamas granuliformes, cuerpo deprimidlo, 4 extremidades, uñas retractiles y dedos en laminillas. (Salamandra).

Gecónidos. (Gecliones).

39 Cuerpo prolongado y protejido por escamas que forman circulo alrederlor del tronco; extremidades cortas ó faltan completamente. (Culebrita ciega). Anfisbénidos. (Amphisbaesca).

Fu. 4. Cocodrmos. (Crocodiline). No tiene representante. en P. R.

Fr. 5. Lacerribos. (Lacertimi).

Ameiva Plei. Dum. Iguana. Una sola especie en P. R.. muy grande, oscura con puntos y cuadros negros y blancos. En los arenales y pedregales.

Fu. 6. I(tuAnidos. (Iyuunoidei).

Un solo genero con varias especies en P. R. Lagarto. (Anolis)

A. velifer. Cuv. Lagarto Camaleón. Verde con manchas oscuras y vientre amarilloso; el $\hat{o}$ con cresta en el dorso; cambia sus colores, como sus congéneres.

A. Gundluchi. Pet. L. de Gundlach. Lomo oscuro. Prentit claro, cabeza mosqueada; el mayor de nuestros lagartos ordiuarios.

A stratulus, Cope. L. tendido. Color del anterior, en el lom varias manchas negras transversales.

A. cristatellus. Bib. L. de cresta. Color de los anteriores con faja clara á lo largo del lomo, con puntos negros al costado de esta faja.

A. pulchellus, Bib. L. hermoso. Muy esbelto; parte superior gris, la inferior blanca y garganta rojo amarilla.

A. Krrugii. Pet. L. de Krug. No conozco aún esta especie.

Fl. 7a Camaleonidos. (Chamaeleonidei).

No tiene representantes en P. $R$.

FL. 8. Esclncidos. (Scincoillei).

Representado en P. R. por la única especie. Lúcia.

(Diploglossus Plei).

FL. 9a (igó́nibok. (Geckonrs). 
Unica especie puerto-riqueña.

FL. 10. Anfisbentoos. (Amphisbaence).

Especie única de P. R. Culebritu ciega, (Amphisbrena caeca). Cuv

ORD. III. Ofrdros. (Ophidia).

Boca pequeña, cabeza no excede en grosor al cuello. Tiflopes.

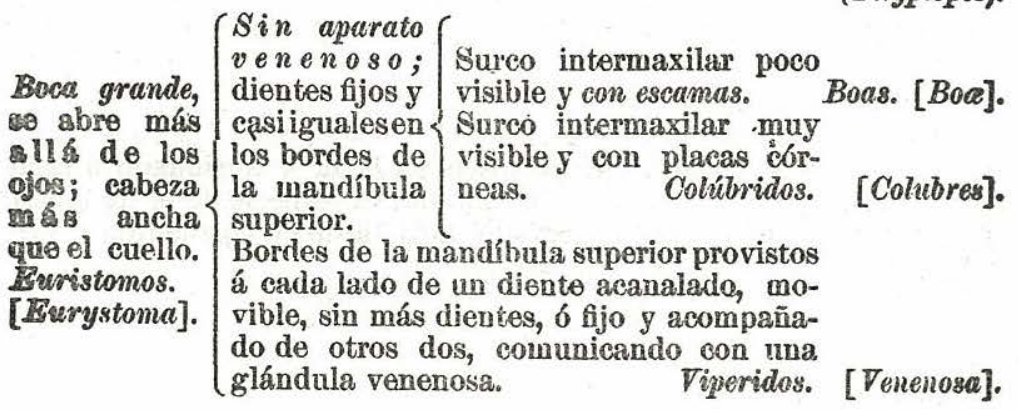

FL. 11. TrFopes. (Typhlopes).

La ánica especie que poseemos. Culebra ciega. (Typhlops lumbiFr. 12. BOAS. (Boa). calis). $\mathbb{L}_{0}$

Un solo género en P. R.

Culebrón. (Chilabothrus).

Ch. inornatus. Reinhardt. Color pardo con cuadros oscuros, viontre más claro.

Fu. 13: Colubridos. (Colubres).

Boca hendida hasta detrás del oido. Culebra sabanera. (Dromicus). Boca hendida hasta el oido. Culebrita. (Alophis).

Dromicus parvifrons. Cope. Oulebra sab. Color canelo oscuro con cuadros negros, vientre blanco.

Alophis portoricensis. Reinhardt. Culebrita. Color pardo claro;

B. Desnudos. (Nuda).

OrD. IV. Batraquios. (Batrachia)

Ff. Anuros. (Anura).

Stb-Fl. Ravas. (Renee). 


\section{(71)}

Solo género y sola especie en P. R.

Sapo. (Cystignathus). бUB-FL. BOMBInATORES.

Solo género y sola especie en P. R. Coqui. (Hylodes.) GUB-Fu, BuFus. (Bufones).

Solo género y sola especie en P. R. Sapo concho. (Bufo). Cystignathus typhonius. Daud. Sapo. Parte superior manchada do oscuro y rojizo, vientre blanco.

Hylodes martinicensis. Thud. Couú. Sobre el espinazo pasa una línea blanca que tiene sus ramales, y el vientre blanco, siendo la parte superior oscura. ancio.

Bufo gutturosus, (Peltaphryne). Latr. Sapo conrho. Amarillo

Don Tomás Blanco, farmacéutico en P. R. y aficionado á la historia natural, ha encontrado allí una nueva especie de la familia de los Geckones que hemos observado más tarde en Aguadilla y otros latgares.

Notes on page 69 :

Line 19: "Ameiva plei" is A. exsul.

Lines 24 et seq. "A. velifer" is A. cuvieri.

"A. gundlachi ... el mayor de nuestros lagartos ordinarios." It is evident that he considered the male of $A$. cristatellus to be $A$. gundlachi. Below where he describes $A$. cristatellus it is obvious that he has the female only in mind.

Next to last line. "Diploglossus plei" is Mabuya nitida. 


\section{APENDICE \\ La Preñez en Ciertos Peces y Reptiles}

Agregamos por vía de apéndice, como conclusiọ́n de la primera parte de la obra, dos curiosas observaciones sobre la preñez en ciertos peces y reptiles.

"Distante estaba de creer que en breve debería hacer aquí la misma observación con nuestra Boa o Culebrón "Chilabothrus inornatus." Esta, el Mraja de Sta. María de la isla de Ouba, "Epicrates angulifer," y algunos otros reptiles, se desvian de la regla ordinaria, y de ovíparos se convierten en vivíparos. Al regresar de Cuba en Junio del próximo año, recibí de regalo de mi amigo D. Tomás Blaneo, aficionado al estudio de la historia natural, un eulebrón hembra que había tenido guardado cerca de un mes, y yo lo dí de compañero â un Majá cubano que guardaba en su buena jaula. Cuál sería mi sorpresa cuando á fines de Setiembre, es decir, $\hat{a}$ los cuatro meses de haber tenido la boa guardada y libre del contacto con el macho, Ia encuentro rodeada de una numerosa prole consistente en 25 hijos recién nacidos y vivos, como de una euarta de largo por un centímetro de ancho, con los colores de la madre, llevando aún trozos del cordón umbilical. En el suelo de la jaula habían varios pedazos más ó menos grandes de una masa consistente, aunque algo blanda y amarilla, parecidas á pedazos de jabon amarillo, probablemente trozos de la placenta."

Note to page 126. Dr. Stahl was not aware that certain reptiles are ovoviviparous. His allusion to a placenta is a slip not to be held against him.

"Pero es el caso, que en los reptiles parece que la regularidad consiste en la irregularidad, así lo prueban las experiencias del Dr. Gundlach cor los huevos del Morrocoyo, $\mathrm{y}$ últimamente la observación hecha por mí con otro culebrón que parió a mediados de Octubre y á los cuatro meses de su cautiverio."

\section{Dr. Francisco del Valle Atiles}

The small brochure by Dr. Franciseo del Valle Atiles is not a copy of Stahl. His systematic classification shows improvement over the earlier writer. He also understood that certain reptiles are ovoviviparous. He omits "Testudo tabulata, Caretta caretta and Diploglossus sagrae" which were included by Stahl and adds Alsophis portoricensis which Stahl had omitted and Hemidactylus brooki which Stahl had alluded to without naming. 
Del Valle names only those animals having a direct bearing on human economy which explains his motive for omitting certain minor species.

The value of republishing this extract from his pamphlet is: $\mathrm{He}$ is the first to mention the food habits of Puerto Rican reptiles which has been much studied of late. He is the first conservationist of the Island. He advocates letting innocent animals live for the pleasure we should receive from knowing them, if not for their inherent right to live.

The following is the title of his pamphlet:

"Los Animales Vertebrados Utiles" y "Los Dañinos a la Agricultura del País," por Francisco del Valle Atiles, Doctor en Medicina y Cirugía.

Memoria, premiada en el Certamen del Ateneo Puertorriqueño, correspondiente al año de 1885, por laudo del Jurado elegido por el Ateneo de Madrid en la Sección de Ciencias Naturales.

Puerto Rico: Imprenta del "Boletín Mercantil", Fortaleza 24 y 26. 1887.

\section{REPTILES}

Es la tercera clase de los animales vertebrados, de generación comúnmente ovípara, suele ser ovovivípara alguna vez; su sangre es fría, la respiración pulmonar, y la circulación incompleta; su alimentación es casi exclusivamente animal, sus dientes parecen destinados más para retener la presa, que por lo común se tragan viva, que para masticar.

Se comprenden en esta clase especies venenosas, como las víboras, el áspid y la serpiente de cascabel, que por fortuna son desconocidas en Puerto-Rico.

Tienen los reptiles una gran resistencia vital, de modo que pueden sufrir grandes mutilaciones y aún algunos poseen la facultad de regenerar las porciones perdidas, en especial la cola. Divídense en cinco órdenes á saber:

Los QUELONIOS que están representados por:

La ToRTuga. Chelonia viridis.-Util por la carne y huevos.

El CAREY. Chelonia imbricata.-Util por su concha, huevos y carme.

El TINGLADo. Sphargis coriacea.-Como el anterior.*

La HicoteA. Emys rugosa.-Util por su carne.

Los emidosauros que comprenden Cocodrilos y Caimanes que afortunadamente no existen en Puer̂to-Rico.

Los enaliosaurios que hoy no existen en el mundo.

Los saurios de los quales se conocen hoy aquí:

El Sphaeriodacdytus macrolepis. Salamandra.-Salamanquesa.

* It is a pity that Dr. del Valle Atiles did not give definite data on this species which would have added another reptile to the Puerto Rico area. There is no definite record of this species having been taken off this Island to the present day. 
El anolis velifer.-Camaleón.

El anolis Gundlachi.

El anolis Cristatellus.

El anolis Stratulus.

El anolis pulchellus.

El anolis Krugii.

Lagartijos.

Todas son útiles á la Agricultura y Horticultura, porque destruyen los insectos.

El Ameiva Plei.-Iguana.-Indiferente como los que siguen:

Euprepes Spilonotus.-Lúcia.

Amphisbaena caeca.-Culebra ciega.

Los ofIDIOs se conocen por:

El Typhlops lumbricalis.-Culebrita ciega.

El Chilabothrus inornatus.-Culebrón.

El Dromicus parvifrons,-Culebra sabanera.

El Alsophis portoricensis.-Culebrita.

Animales estos con los que la preocupación se ha manifestado más rebelde, debemos declararlos inocentes; de ellos el culebrón, que se come algunos huevos y viene á las habitaciones tras las aves domésticas, destruye en cambio muchísimas ratas.

\section{BATRACIOS}

Animales de conformación adecuada para vivir alternativamente en el agua $\delta$ en el aire, tienen algunos respiración branquial y pulmonar á la vez en la edad adulta, mientras que la generalidad respira por branquias en la primera edad y por pulmones en la segunda. La circulación es doble é incompleta.

El aparato digestivo es corto, circunstancia que se explica por que generalmente son Zoofagos. Se alimentan de pequeños moluscos y otros animales pequeños; su reproducción es ovípara.

En Puerto-Rico se han estudiado hasta ahora:

El Cystignathus typhonius.-Sapo.

El Bufo gutturosus.-Sapo concho.

El Hylodes martinicensis.-Coquí.

Estos animales son útiles á la agricultura pues destruyen gran número de insectos, moluscos y caracoles nocivos á los sembrados.

Debe respetarse á estos inofensivos vertebrados que nos reportan utilidad suma. 